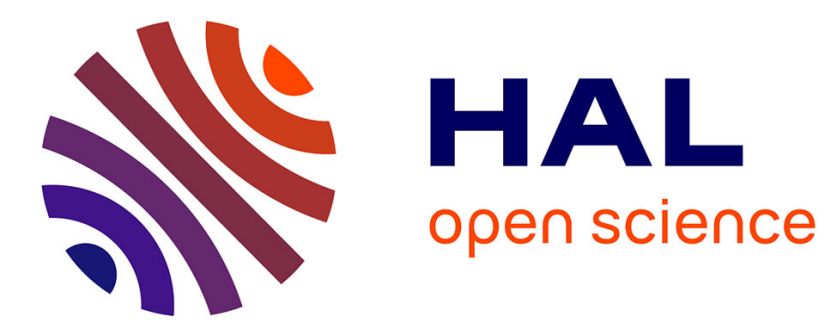

\title{
La critique de la modernité technique : termes et variations
}

\author{
Alexandre Moatti
}

\section{To cite this version:}

Alexandre Moatti. La critique de la modernité technique : termes et variations. Sens-Dessous, 2019, Humanités, 24, pp.97-105. 10.3917/sdes.024.0097 . hal-02301819

\section{HAL Id: hal-02301819 \\ https://hal.science/hal-02301819}

Submitted on 30 Sep 2019

HAL is a multi-disciplinary open access archive for the deposit and dissemination of scientific research documents, whether they are published or not. The documents may come from teaching and research institutions in France or abroad, or from public or private research centers.
L'archive ouverte pluridisciplinaire HAL, est destinée au dépôt et à la diffusion de documents scientifiques de niveau recherche, publiés ou non, émanant des établissements d'enseignement et de recherche français ou étrangers, des laboratoires publics ou privés. 


\section{humanitēs 24}

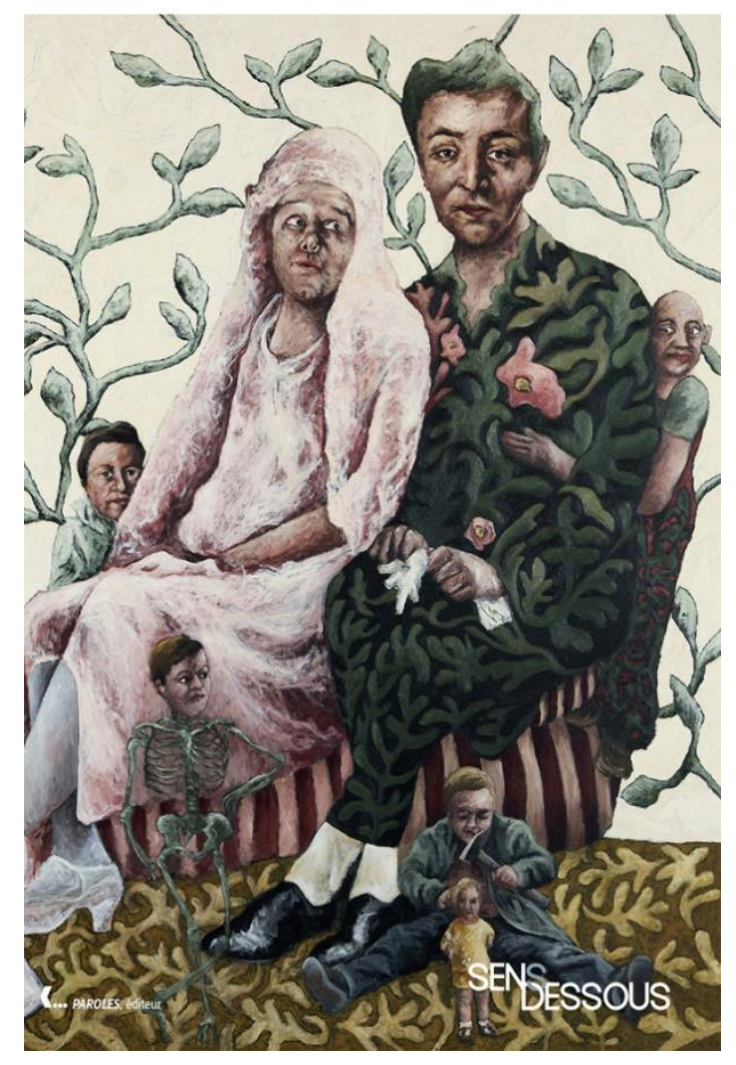

Moatti Alexandre, «La critique de la modernité technique : termes et variations », Sens-Dessous, 2019/2 (N²4), p. 97-105. DOI : 10.3917/sdes.024.0097.

Sommaire complet à : https://www.cairn.info/revue-sens-dessous-2019-2.htm Éditrice de la revue : Mme Nadia Taïbi (et Association Paroles) 


\section{La critique de la modernité technique : termes et variations}

L'histoire de la critique de la modernité technique - notre sujet - s'adjoint utilement celle du vocabulaire critique. Elle amène en particulier à rencontrer certains néologismes ou mots fort peu usités, en support de la rhétorique argumentaire : c'est sans doute le cas dans tout domaine de recherches, au moins en histoire des idées, mais peut-être plus particulièrement en ce qui concerne l'étude d'une critique, quelle qu'elle soit. D'ailleurs la critique de la modernité - qu'Antoine Compagnon a baptisée celle des Antimodernes ${ }^{1}$ - aurait selon cet auteur une caractéristique (avec cinq autres) : la vitupération. On peut en dire là aussi tout autant de toute forme de critique... Disons alors que repérer ces néologismes, ces expressions-choc est une des méthodes de travail et d'analyse du chercheur en la matière.

Comme l'est l'histoire des mots plus courants (ceux qui ne sont pas des néologismes à usage rhétorique), qui est au cœur de l'histoire des idées - leur apparition, leur période de «mode», leur déclin. Par exemple : quand apparaît le mot transhumanisme ${ }^{2}$ ? Le mot machinisme - aujourd'hui un peu désuet - connaît son heure de gloire dans les années 1930, utilisé par divers courants de pensée (notamment le personnalisme et les idéologies de $3^{e}$ voie) très réticents à une société organisée et taylorisée - dans la vie personnelle comme la vie professionnelle, au bureau, à l'usine - modèle de société venant tout droit des États-Unis et dénoncé comme tel. Ce qui s'est appelé la « querelle du machinisme» apparaît dans un outil qui nous est donné par les technologies numériques :

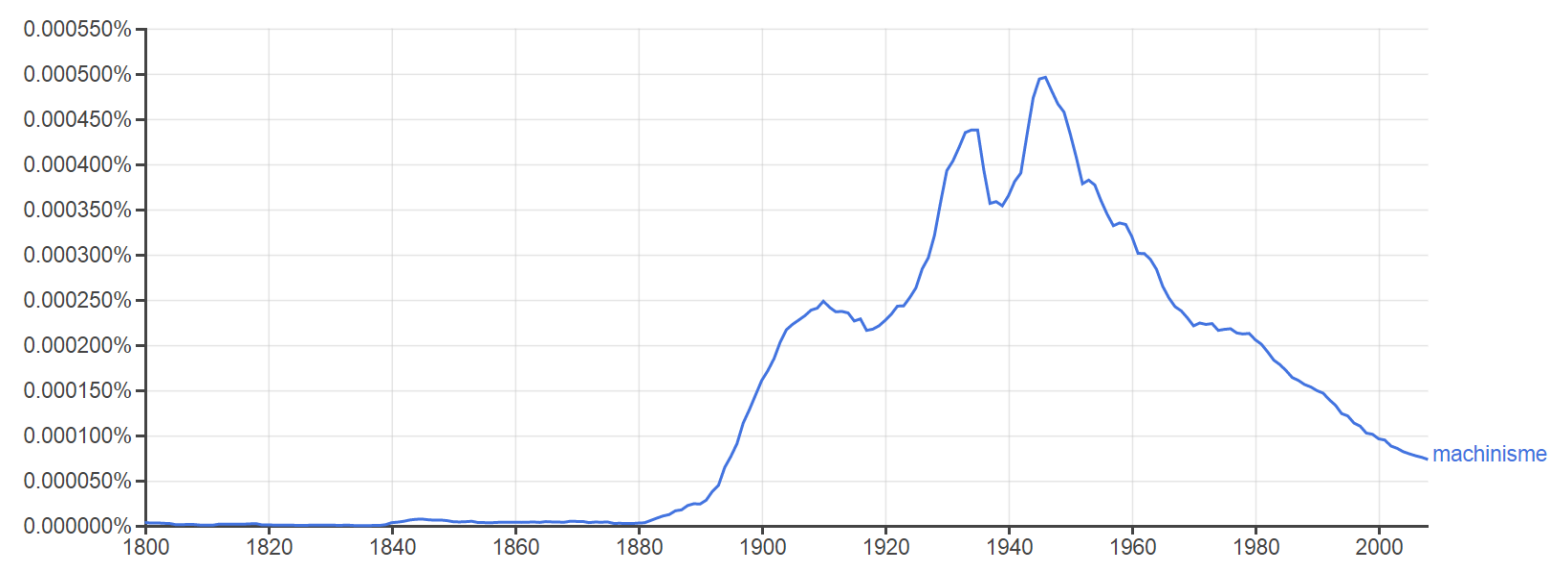

Évolution des occurrences du terme machinisme entre 1800 et 2000. La pointe des années 1930 correspond à la « querelle du machinisme ». Si cette analyse, réalisée

\footnotetext{
${ }^{1}$ Antoine Compagnon, Les Antimodernes. De Joseph de Maistre à Roland Barthes, Gallimard Idées, 2005.

2 Olivier Dard \& Alexandre Moatti, « Aux origines du mot transhumanisme », Futuribles, n413, juillet-août 2016 [version d'origine, plus courte, en anglais : " The history of transhumanism (cont.) ", Notes and Queries, 2017, 262 [New Series, 64], pp.167-170, Oxford University Press.
} 
avec Google Ngram Viewer, peut être biaisée car fondée sur la base des ouvrages numérisés par Google, la tendance lourde ne trompe pas : l'utilisation du mot est multipliée ${ }^{3}$ par 100 entre 1870 et 1940.

Cependant le mot machinisme a une histoire plus ancienne : il est proposé par un auteur qu'on n'attendait pas forcément là, l'historien Jules Michelet (1798-1874), dans Le Peuple ${ }^{4}$ en 1846 (c'est la petite bosse qu'on voit à cette époque dans le diagramme cidessus, qu'on pourrait zoomer) :

Cette malheureuse population asservie aux machines comprend quatre cent mille âmes, ou un peu plus (*).

$[\mathrm{NbdP}]\left({ }^{*}\right)$ - Ceux qui étendent ce chiffre y comprennent des ouvriers occupés, il est vrai, dans des manufactures qui emploient des machines. Ceux-ci sont et seront toujours une exception. - L'extension du machinisme (pour désigner ce système d'un mot) est-elle à craindre ? La machine doit-elle tout envahir ? La France deviendra-t-elle sous ce rapport une Angleterre? - A ces questions graves, je réponds sans hésiter : Non.

Mais Michelet donne - en complément - une acception bien plus vaste et allégorique du terme. Avec un grand M (le Machinisme, toujours dans Le Peuple, p. 123), il en appelle à l'Histoire pour montrer qu' après la Renaissance et l'épanouissement des arts, la société a regrettablement cherché à s'organiser : " on refit des machines administratives, bureaucratie à la Colbert, armées à la Louvois », « le Machinisme [a] mécanisé le monde ailé de la fantaisie ». C'est même un « machinisme administratif, industriel, politique, littéraire ", ces deux derniers s'attaquant à la " méditation philosophique » qui est le propre de l'Homme. Dès $1843^{5}$, Michelet s'était élevé contre un « machinisme moral »: « au milieu d'un immense accroissement de production matérielle, la production intellectuelle a considérablement diminué d'importance », on ne fait que des « réimpressions de livres pittoresques à bon marché »- on trouve là une critique, qu'on retrouvera souvent par la suite, de l'essor de la technique au

\footnotetext{
${ }^{3}$ L'outil est construit correctement : «l'utilisation » est mesurée par le pourcentage d'occurrences à une date donnée, dans le corpus d'ouvrages numérisés. C'est donc une valeur relative, la seule valable (ainsi, dans ce cas, l'occurrence du terme machinisme passe de 5 livres par million de livres à 5 par 10000 livres...)

${ }^{4}$ La suite de la note de bas de page est faite de considérations qui ne seront pas vérifiées (preuve que l'historien a du mal à faire de la prospective) : « plus les premiers besoins seront satisfaits à bas prix par les machines, plus le goût s'élèvera au-dessus des produits du machinisme, et recherchera les produits d'un art tout personnel », en ce dernier point « est le véritable avenir de la France industrielle, bien plus que dans la fabrication mécanique où elle reste inférieure [à l'Angleterre] ".

${ }^{5}$ Ceux qui s'intéressent plus avant à Michelet peuvent consulter cet article de référence de Paul Villaneix dans la revue Romantisme en 1979 (en ligne sur Persée). L'auteur, grand spécialiste de Michelet, atteste dans cet article le premier emploi du mot machinisme en 1843.
} 
détriment des savoirs. Le feuilleton rétrospectif n'est pas terminé, puisqu'en fait le mot machinisme existait au XVIII ${ }^{\mathrm{e}}$ siècle dans un sens différent - celui du machinisme au théâtre, à l'opéra (contre lequel certains puristes de la scène s'élevaient, aussi...). De nos jours ce sens existe toujours - et surtout : un machiniste de théâtre, comme pour un conducteur d'engins et de machines (dans le bus « il est [ou était] interdit de parler au machiniste »).

\section{*}

Un autre terme est au cœur de notre thème de recherches : c'est bien sûr la modernité. Là encore l'outil Ngram Viewer est d'une redoutable efficacité :

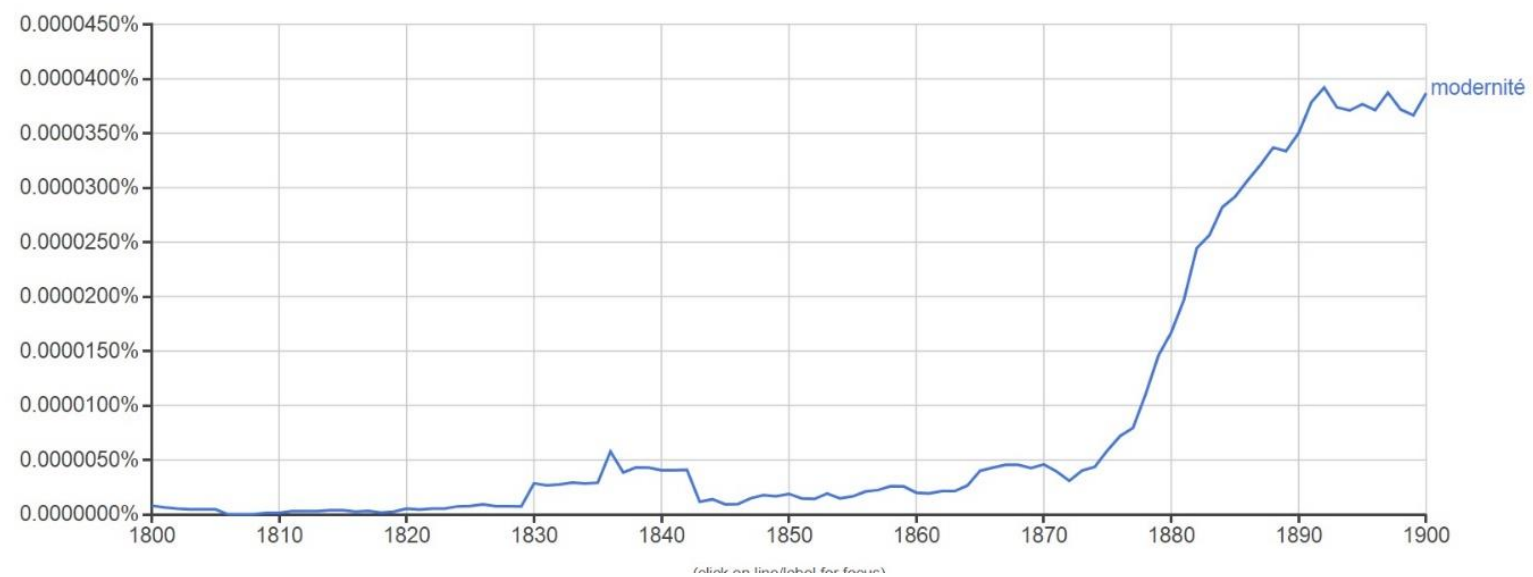

Évolution des occurrences du terme modernité au XIXe siècle. Sur une bien plus brève période de temps que dans le graphique précédent, l'utilisation du terme est multipliée par sept entre 1875 et 1890.

Le terme modernité est, au cours du XIXe siècle, d'abord lié à la littérature et à l'art (les Anciens et les Modernes, toujours en querelle); il apparaît plus tardivement comme lié à la technique. À partir du graphique ci-dessus, qui atteste d'une quasi rupture sémantique, on peut même émettre l'hypothèse que le terme serait constitutif de la III République et de son positivisme triomphant, et même plus précisément de la III République enfin installée, à la fin des années $1870^{6}$.

De quoi donner raison rétrospectivement avec NGram Viewer à... Charles Péguy, un des premiers « antimodernes » (selon Compagnon), en tout cas chantre critique de la modernité technique, qui voyait le «monde moderne», celui « qui fait le $\operatorname{malin}^{7}$ »,

\footnotetext{
${ }^{6}$ Nous avons développé cette idée, et avec elle celle d'une technocratie avant la lettre comme elle aussi constitutive de la modernité d'une France enfin républicaine dans "Adolphe Alphand : quelles traditions polytechniciennes ? ", in M. Audouy, J.-P. Le Dantec, Y. Nussaume, C. Santini (dir.), Le Grand Pari(s) d'Alphand. Création et transmission d'un paysage urbain, Éditions de la Villette, 2018.

${ }^{7}$ Ch. Péguy, Notre jeunesse (1910), Folio Essais, Gallimard, 1993, p. 102.
} 
commencer «en 1881 » - Péguy, d'ailleurs grand admirateur de Michelet dans la lignée des historiens, contre Renan, contre Taine... Ce qui nous amène à notre seconde partie, celle des néologismes ou des mots inusités, utilisés pour renforcer une critique de la modernité technique. Péguy en effet, avec son style si particulier - vitupérant $c^{\prime}$ est certain -, constitue une mine de tels mots et expressions. Un morceau de bravoure est la tirade du «sac à dos scientifique », celui des armées modernes (1907, par opposition à celui de l'An II) :

[...] leur sac n'était point cet énorme sac moderne raide des armées modernes, ce sac scientifique, où l'on a tout mis, tout prévu, excepté, ce sac ennemi de l'homme, scientifiquement fait, scientifiquement établi, scientifiquement construit, scientifiquement imaginé, où l'on a scientifiquement tout mis, scientifiquement tout prévu, hormis, qu'on a scientifiquement oublié d'y mettre ceci : que l'homme aurait envie de le porter ; de l'avoir, de le garder, sur ses deux épaules, de marcher dessous ; sac moderne, ennemi de l'homme, où scientifiquement et modernement on a tout prévu, excepté, scientifiquement et modernement, un petit coin dedans, bien abrité, pour y mettre la bonne volonté $[\ldots]^{8}$

Dans la plus pure rhétorique péguyste, on voit donc l'adverbe scientifiquement figurer une dizaine de fois en quelques lignes. Y figure aussi l'adverbe MODERNEMENT, mot certes répertorié (p. ex. dans les Mémoires du duc de Saint-Simon, et aussi à la fin du XIXe s.), mais fort peu répandu. Le substantif MODERNIANISME est, lui, un néologisme créé de toutes pièces par Péguy et utilisé par lui uniquement, parlant de Paris, capitale «[d]u modernianisme, rendez-vous de toutes les hérésies ${ }^{9}$ »; le modernianisme, c'est l'idéologie de la modernité. On trouve aussi quasi exclusivement chez Péguy l'adjectif SCIENTIFICISTE ${ }^{10}$, comme synonyme de positiviste ou de scientiste, mais utilisé pour décrire avec une ironie affectueuse son ami Bernard Lazare.

On connaît enfin l'inimitié de Péguy contre le " parti intellectuel », contre la sociologie universitaire naissante, accusée de déformer la réalité en une matière à étude, de transformer la mystique en politique - cette pédagogie, cette sociologie, condensées dans le mot-valise de SOCIAGOGIE :

(que nous nommerons désormais, je vous préviens, sociagogie, parce que c'est beaucoup mieux), démagogie et autres agogies qui ont entrepris d'exercer [...] une tyrannie mentale, intellectuelle, morale, civique $[\ldots]^{11}$

\footnotetext{
${ }^{8}$ Ch. Péguy, Cahiers de la Quinzaine, IX-1 du 6 octobre 1907, « De la situation faite au parti intellectuel dans le monde moderne devant les accidents de la gloire temporelle ».

${ }^{9}$ Ch. Péguy, " De la situation faite au parti intellectuel dans le monde moderne [...] », Cahiers de la Quinzaine, IX, 1 (octobre 1907), in Pléiade, CEuvres en prose, 1898-1908, éd. M. Péguy, 1959, p. 1176.

${ }^{10}$ Ch. Péguy, Notre jeunesse (1910), Folio Essais, Gallimard, 1993, p. 174.

${ }^{11}$ Ch. Péguy, Cahiers de la Quinzaine, VIII, 11 (in Pléiade éd. Burac vol. 2, p. 642 et ss)
} 
Et, dans la même veine, le substantif ou adjectif sOCIOLOGICABLE - ce qui est susceptible de devenir sociologie, Péguy déplorant « un monde presque uniquement sociologicable, je veux dire un monde qui n'est presque bon à rien, qu'à être mis en sociologie ${ }^{12} \gg$.

Dans ces déclinaisons du sociologique, on trouvera un auteur inattendu ici : quoique vitupérant (peut-être son seul point commun avec Péguy), il était loin d'être un contempteur de la modernité technique - ceci dit, il existe bien des points communs entre exaltation et critique de la modernité ${ }^{13}$. Je parle du philosophe Auguste Comte (1798-1857) : certains de ses disciples en ont même fait le symbole du gouvernement par la science. On le donne généralement comme inventeur du mot et du concept de sociologie. Il utilise aussi sociocratie, mot qui a connu une seconde jeunesse dans les années 1950-1960. Mais ce qui nous intéresse ici est un terme uniquement comtien, la SOCIOLÂTRIE ${ }^{14}$ : dans la religion positiviste de l'Humanité qu'il fonde - après avoir été le philosophe des sciences que l'on connaît le plus souvent - c'est le culte du GrandÊtre, le culte d'une société régénérée à travers celui-ci, de fait une idolâtrie revisitée,

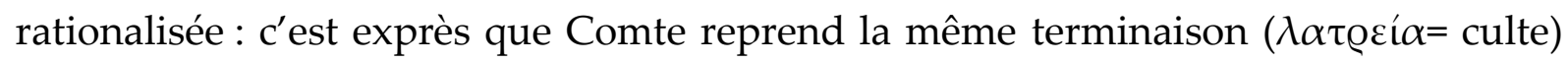
que le terme idolâtrie, la religion positiviste se calquant sur de nombreux points sur les religions révélées et notamment le christianisme. Et le calendrier de cette religion, composé de treize mois de 28 jours, s'appelle le «tableau sociolâtrique de l'Humanité ${ }^{15} »$.

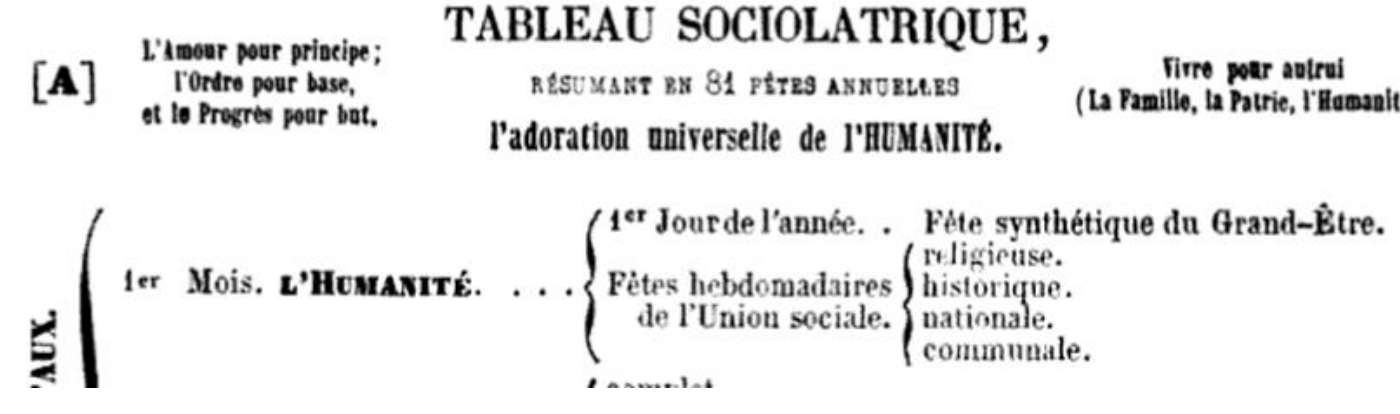

A. Comte, Système de politique positive, t. IV, p. 159, extrait

\footnotetext{
${ }^{12}$ Ch. Péguy, Brunetière, 1906, inédit (in Pléiade éd. Burac, vol. 2, p. 626). Sur Péguy et la sociologie, un colloque 'Péguy chantre de l'action sociale et pourfendeur des sociologues' s'est tenu en décembre 2016 (Amitié Charles Péguy, en partenariat avec le CELLF (UMR 8599, Université Paris-Sorbonne; https://www.fabula.org/actualites/colloque-peguy-chantre-de-l-action-sociale-et-pourfendeur-dessociologues 76839.php)

${ }^{13}$ Voir à ce propos l'argumentaire développé dans $A$. Moatti, « Pour une critique raisonnée de la technique et de I'Internet ", Commentaire, n 154, été 2016 (en ligne HAL-SHS).

${ }^{14}$ Nombreuses occurrences, par exemple dans le Système de Politique positive, 1851-1854, t. IV (27 occurrences dans ce tome).

${ }^{15}$ Système de politique positive, t. IV, op. cit., p. 159. Cité par Bernard Guillemain dans la notice de l'Encyclopedia Universalis consacrée à Comte. L'auteur donne comme exemple: "Innocent III est fêté le 21 Charlemagne (cinquième mois) et saint Thomas d'Aquin le 7 Descartes (onzième mois) [...] ", et ajoute, non sans humour : "Commenter serait délicat. »
} 
Revenons aux néologismes descriptifs d'une idéologie, tel le modernianisme chez Péguy. On trouve un néologisme utilisé dans les années 1960, notamment dans le roman de Georges Perec, Les Choses (1965) : le TECHNOCRATISME, «culte de l'efficience, de la modernité », à propos du jeune couple héros du roman, entièrement soumis à la société de consommation :

Dans les milieux de la publicité, généralement situés, d'une façon quasi mythologique, à gauche, mais plus aisément définissables par le technocratisme, le culte de l'efficience, de la modernité, de la complexité, de la spéculation prospective, la tendance plutôt démagogique à la sociologie, et l'opinion, encore assez répandue, que les neuf dixièmes des gens étaient des cons tout juste capable de chanter en chœur les louanges de n'importe quoi ou de n'importe qui $[\ldots]^{16}$

On connaît bien sûr - ô combien ! - la technocratie, le technocratisme serait l'idéologie véhiculée par les technocrates, ou le comportement technocratique. Le terme est rare, et semble faire florès dans une période allant de quelques années avant à quelques années après 1968, ce principalement dans un milieu universitaire ou savant - milieu que ne dépréciait pas Perec. Il est utilisé fréquemment et précocement, dès 1951, par le sociologue Pierre Fougeyrollas (1926-2008), qui dans un pesant article marxiste l'emploie à plusieurs reprises, par exemple : «Le taylorisme est une organisation du travail par en haut, un technocratisme en vue du profit patronal ${ }^{17}$. » Il est utilisé dans un article beaucoup plus intéressant des Temps Modernes en 1966, mystérieusement anonyme, ce qui est rarissime dans ce type de revues : l'article analyse les livres des patrons technocrates Louis Armand, ingénieur des Mines ${ }^{18}$, et François Bloch-Lainé, inspecteur des Finances ${ }^{19}$; à leur propos, il indique que le technocratisme est une idéologie qui «se baptise structurellement toujours nouvelle»; "ce que le technocratisme passe son temps à définir, c'est lui-même comme différent du capitalisme $^{20}$. » Et en 1972, Deleuze l'utilise dans un sens analogique (de " pouvoir », sens plus indifférencié, où il perd en précision) : «C'est curieux que l'épistémologie ait

\footnotetext{
${ }^{16}$ G. Perec, Les Choses. Une histoire des années soixante, 10/18, René Julliard, 1965, p. 71-72.

${ }^{17}$ P. Fougeyrollas, "De la psychotechnique à la sociologie policière ", La Nouvelle Critique, revue du marxisme militant, $3^{\mathrm{e}}$ année, $\mathrm{n}^{\circ} 28$, juillet-août 1951, p. 25-46.

${ }^{18} \mathrm{~L}$. Armand, Plaidoyer pour l'avenir (en collaboration avec Michel Drancourt), 1961. Sur cet auteur et sa pensée managériale, voir Moatti, Alexandre. « Vocabulaire et controverses autour de la cybernétique et du transhumain, années 1960-1970 ", L'Homme \& la Société, vol. 205, no. 3, 2017, pp. 109-131.

${ }^{19} \mathrm{~F}$. Bloch-Lainé, Pour une réforme de l'entreprise, Le Seuil, 1963.

${ }^{20}$ XXXX, « L'idéologie technocratique et le teilhardisme », Les Temps Modernes, n²43, 1966, p. 254-295. L'article analyse aussi comment " le tourbillon dans lequel s'enroule le technocratisme est le teilhardisme » (du nom du R.P. Teilhard de Chardin, 1881-1955 et de ses doctrines). Dans Moatti, "Vocabulaire et controverses... ", supra, j'analyse l'invocation de Teilhard par Louis Armand, ce que fait l'article des Temps Modernes, à l'époque en 1966, de manière brillante et approfondie (je n'ai découvert que plus tard cet article).
} 
toujours caché une instauration de pouvoir, une organisation de pouvoir, une sorte de technocratisme universitaire ou idéologique ${ }^{21}$. » Le terme est tombé en désuétude : on pourrait néanmoins se demander si la technocratie élevée au rang de politique, que l'on connaît en France depuis 2017, ne justifierait pas de relever le concept de technocratisme - qui en première approche paraît redondant par rapport à technocratie, mais qui finalement va plus loin et apporte une précision à ce terme: le technocratisme, c'est l'idéologie de la technocratie.

Remontons jusqu'à Baudelaire - lui aussi critique de la modernité s'il en fût, lui aussi « antimoderne » - et l'un de ses mots forgés ad hoc, zOOCRATE (1855) :

Demandez à tout bon Français qui lit tous les jours son journal dans son estaminet, ce qu'il entend par progrès, il répondra que c'est la vapeur, l'électricité et l'éclairage au gaz, miracles inconnus aux Romains, et que ces découvertes témoignent pleinement de notre supériorité sur les anciens ; [...] Le pauvre homme est tellement américanisé par ses philosophes zoocrates et industriels, qu'il a perdu la notion des différences qui caractérisent les phénomènes du monde physique et du monde moral, du naturel et du surnaturel ${ }^{22}$.

Le philosophe zoocrate, c'est celui qui exerce son pouvoir et son magistère par les lois de la nature : c'est celui qui pratique la philosophie naturelle (c'est ainsi qu'on appela longtemps la physique), c'est-à-dire, en gros, le scientifique. Le mot est assez pompeusement redéfini dans des blogs contemporains ${ }^{23}$ - preuve de sa « modernité » là aussi -, comme s'il était inventé par les auteurs de ces sites !; alors que Baudelaire, lui, n'avait pas besoin de définir ce néologisme, supposé compris par son lecteur d'ailleurs ce n'était pas vraiment son style que donner des définitions. À propos de contemporanéité, je ne résiste pas à l'envie de décrire la définition que me donna un de mes étudiants de M1, dans un cours où, faisant étudier ce passage de Baudelaire, je laissais deviner le sens du terme : "ah, monsieur, zoocrate c'est le pouvoir exercé à l'époque par les industriels sur les ouvriers - ils les considéraient comme des

\footnotetext{
${ }^{21}$ "Deleuze et Guattari s'expliquent », La Quinzaine littéraire, n 143, 1972, p.17 (cet article est une joute à plusieurs à propos de l'ouvrage de Gilles Deleuze \& Félix Guattari, L'Anti-CEdipe, Minuit, 1972).

${ }^{22} \mathrm{Ch}$. Baudelaire, Critique d'art, "Exposition universelle - 1855 - Beaux- Arts; I. Méthode critique de l'idée moderne de progrès appliquée aux Beaux-Arts. Déplacement de la vitalité » (1855), Folio Essais, Gallimard, 2005, p. 240.

${ }^{23} \mathrm{Ex}$ : « J'appelle ici zoocratie, la nouvelle idéologie qui, sous prétexte de ne voir l'homme que comme produit de la nature, le réduit à une animalité sans les au-delà métaphysiquement admis qui l'ont toujours caractérisé comme humain et donc humainement inclassable au niveau naturel. », Camille Loty Malebranche, 25 avril 2014, http://intellection.over-blog.com/article-de-la-zoocratie-ideologique-contemporaine-81002132.html
} 
animaux. » Une interprétation amusante, en tout cas originale, que peut-être Baudelaire n'aurait pas reniée.

À la fin du XIXe siècle, dans le cadre de la philosophie naturelle justement - la biologie, cette fois-ci - apparaît un incroyable terme, dans le sillage de Darwin: le STRUGGLEFORLIFISME (mot français, de l'anglais struggle for life, combattre pour la vie combat censé dériver de la théorie darwinienne de la sélection naturelle, appliquée à l'homme). Encore une fois preuve que le français, et le Français, adorent les termes en -isme, puisque le terme équivalent (struggleforlifism) n'existe pas en anglais, la langue de Darwin! Jean-Marc Bernardini, spécialiste de la réception du darwinisme en France, explique même la lutte entre deux types de struggleforlifisme, évoquant un auteur de 1901, qui « s'opposait au "struggleforlifisme individuel et antisocialiste" au profit d'un " "struggleforlifisme collectif" et d'un lamarckisme social plus compatible avec un égalitarisme social et des idées de progrès et d'évolution par l'éducation ${ }^{24}$ ». Donc deux types de struggleforlifismes combattaient entre eux, tels des concepts soumis à la sélection darwinienne; et surtout la France, après avoir imaginé ce néologisme, l'adaptait à son lamarckisme très prégnant (et en 1900 mâtiné de radicalsocialisme) - toujours et encore cette adaptation de Darwin !, laissant l'acception originelle au libéralisme britannique.

On retrouve le terme, qui fleure bon ces joutes écrites de la III ${ }^{e}$ République, dans un tout autre contexte, presque surréaliste - le Colloque abhumaniste qu'entretiennent les deux auteurs Jacques Audiberti et Camille Bryen en 1952. L'abhumanisme, c'est la nécessité de s'extraire de la vision d'un homme tout-puissant (par la science et la technique notamment); ce colloque est une belle page de critique de la modernité technique au sortir de la Seconde Guerre mondiale. Est-ce intentionnellement que les deux auteurs, facétieux, ressortent ce vieux terme? :

Hélas! Fourrer dans le même sac l'élan de l'âme et le saut de la panthère, c'est faire profession de brutalisme, de mécanisme, de matérialisme, de struggleforlifisme. Ce sont des vocables qui ne veulent rien dire, hormis, bien entendu, eux-mêmes ${ }^{25}$.

\section{*}

Ce terme d'aвнUмANISME chez Audiberti et Bryen nous amène enfin, pour conclure, au thème principal de ce numéro. On pourrait considérer, en première approche, le terme abhumanisme audibertien de 1952 comme un antonyme complet au transhumanisme contemporain. André Breton ne proposait-il pas, dès 1924,

\footnotetext{
${ }^{24}$ Jean-Marc Bernardini, Le Darwinisme social en France (1859-1918). Fascination et rejet d'une idéologie, CNRS Editions, 1997.

${ }^{25}$ J. Audiberti et C. Bryen, L'Ouvre-boîte. Colloque abhumaniste, 1952 ; rééd. Les presses du réel, Dijon, 2018, p. 22.
} 
$\mathrm{d}^{\prime}$ " échapper, dans la mesure du possible, à ce type humain dont nous relevons tous », en ajoutant « voilà tout ce qui me semble mériter quelque peine ${ }^{26}$ » ? L'abhumanisme permettrait $\mathrm{d}^{\prime}$ « en finir enfin avec l'Homme roi de l'univers et dominateur, reclasser l'être humain comme un élément du $\operatorname{cosmos}^{27} »$; contre l'humanisme, vu comme dessein programmatique ayant conduit à Hiroshima, Audiberti propose juste aprèsguerre un

«hommisme », c'est-à-dire le fait et l'état directs de l'homme, alors que l'humanisme représente l'homme à un degré programmatique, secondaire et pour ainsi dire professionnel ${ }^{28}$.

Le vrai humaniste, c'est l'abhumaniste, celui qui renoncé à la suprématie de l'homme dans la nature et le cosmos, notamment vis-à-vis de l'animal - on est assez loin cependant, nous semble-t-il, de l'antispécisme actuel, et plus proches d'une forme de bestiaire surréaliste visant à renforcer l'idée de s'extraire d'un humanisme conventionnel et compassé.

Mais si l'on veut voir l'abhumanisme comme antonyme du transhumanisme contemporain, les choses se compliquent en seconde approche. D'abord Audiberti n'est pas un contempteur absolu de la science : selon le duo d'auteurs, la science est capable de produire aussi une poétique, elle réenchanterait le monde - et les savants « ouvrent la porte à la rafale abhumaniste, sans apparemment le vouloir ${ }^{29}$ ». Par ailleurs, les transhumanistes contemporains - ou certains d'entre eux - opèrent une forme de décentrement par rapport à l'homme, se rapprochant de mouvements antispécistes ( «il faut rejeter ce "préjugé humain” et la morale humaniste conservatrice qu'il inspire $\left.^{30} »\right)$, prônant «le respect des êtres vivants non humains et, anticipativement, le respect d'êtres futurs plus ou moins différents des humains actuels $^{31} »$ :

Le préfixe «trans» dans «transhumanisme» est un vecteur qui communique de $l^{\prime}$ humanité vers $l^{\prime}$ « infra-humain » autant que vers le « supra-humain ${ }^{32}$.

\footnotetext{
${ }^{26}$ Les Pas perdus, Gallimard, 1924 [rééd. Gallimard Idées, 1970, p. 14]

${ }^{27}$ Michel Giroud, préface à la réédition de L'Ouvre-boîte, op. cit., p. 8.

28 J. Audiberti, préface à B. Joppolo, Les Chevaux de bois, Gallimard, 1947.

${ }^{29}$ L'Ouvre-boîte (rééd.), op. cit., p. 40.

${ }^{30}$ G. Hottois, Philosophies et idéologies post/transhumanistes, Vrin, 2017, p. 119, examinant la doctrine du philosophe Julian Savulescu (né en 1963). Nous avons une certaine proximité d'approches avec le philosophe belge Gilbert Hottois (1941-2019), qui s'est lui aussi beaucoup intéressé à l'histoire et au sens des termes dans l'analyse de la critique et de l'exaltation de la modernité technique (et particulièrement, pour cette dernière, le transhumanisme).

${ }^{31}$ Hottois, op. cit. p. 121.

32 Hottois, op. cit. p. 121.
} 
L'abhumanisme audibertien ne rejetant pas tout dans la science et la technique, qui fondent le transhumanisme contemporain ; celui-ci, attrape-tout (y compris la doctrine antispéciste), opérant un décentrement, une extraction (limitée), de sa position traditionnelle de l'homme-roi, de l'homme-univers : voilà deux éléments qui rendent difficile l'idée d'un abhumanisme antonyme complet du transhumanisme. Sans doute ces deux concepts continueront-ils à jouer à cache-cache, parfois à 1..2..3...soleil, tout en poursuivant leurs deux routes, fort différentes il est vrai.

Notre conclusion nous amènera à un dernier terme, toujours en anthropologie, au sens de philosophie de l'homme et de l'humanisme : c'est le terme inventé par le sociologue et philosophe Henri Lefebvre en 1967, le CYBERNANTHROPE. Dans son remarquable ouvrage Position. Contre les technocrates, il fait apparaître cette nouvelle forme d'humains - ils sont déjà là, parmi nous, comme les rhinocéros d'Ionesco qui font l'image de couverture de l'ouvrage. Ce sont des technocrates, ou ceux que les technocrates inspirent ou dirigent. Ils connaissent les défauts de l'homme - leurs propres défauts - et veulent y remédier, en reniant ce qu'il y a d' " humain » en eux. Ils ont complétement intégré dans leur comportement les concepts de stabilité et d'auto-régulation, chers à la cybernétique d'alors - mais tout ceci est aisément transposable à nos jours, non?

Le plus intéressant dans l'analyse de cette nouvelle espèce humaine est le combat que Lefebvre met en scène entre celle-ci et l'homme résiduel, le non-mutant, qu'il qualifie non plus d'humain mais d'Anthrope ${ }^{33}$, par opposition au cybernanthrope. Il dramatise ce combat, un peu comme s'il s'agissait d'envahisseurs : « les apparences peuvent être trompeuses ", tel bon vivant qu'on qualifierait volontiers d'anthrope a en fait une vie réglée et instituée - c'est un cybernanthrope. Ce combat sera long et difficile. Les cybernanthropes «se veulent, se disent, se font planétaires et mondiaux». Mais les anthropes ont pour eux «l'imprévisible », "l'irréductible », ainsi que «l'ironie », « le sens du cocasse » - cocasse que l'anthrope perçoit chez le cybernanthrope, trop sérieux, trop engoncé dans « la raison, la science, le plan, le programme, la cybernétique ${ }^{34}$ ». La guerre des anthropes contre les cybernanthropes «sera une guérilla ». Lefebvre en imagine le dénouement en concluant son ouvrage par une note finalement optimiste : "L'anthrope devra savoir qu'il prescrit une manière de vivre plus qu'une théorie philosophico-scientifique [...].» Sa dernière phrase sera la nôtre : elle justifie nos recherches sur la terminologie critique de la modernité technique, notre souci à les présenter et à la rhétorique de ses auteurs : en référence et en hommage à Péguy, à

\footnotetext{
33 Ibid., p. 221.

${ }^{34}$ Ibid., p. 228.
} 
Audiberti - et peut-être même à Comte ! - nous conclurons avec Lefebvre en nous plaisant à croire que - seule arme possible - : « $1^{\prime}$ Anthrope vaincra par le Style ${ }^{35}$ ».

Alexandre MOATTI est ingénieur en chef des Mines, chercheur associé en histoire des sciences au laboratoire SPHERE (UMR 7219), université Paris-Diderot.

${ }^{35}$ Ibid., p. 230. 